\title{
Adaptive and Innate Immune Responsiveness to Borrelia burgdorferi sensu lato in Exposed Asymptomatic Children and Children with Previous Clinical Lyme Borreliosis
}

\author{
Barbro H. Skogman, ${ }^{1,2}$ Sandra Hellberg, ${ }^{3}$ Christina Ekerfelt, ${ }^{3}$ Maria C. Jenmalm, ${ }^{3}$ \\ Pia Forsberg, ${ }^{4}$ Johnny Ludvigsson, ${ }^{5}$ Sven Bergström, ${ }^{6}$ and Jan Ernerudh ${ }^{3}$ \\ ${ }^{1}$ Department of Pediatrics, Falun General Hospital, 79182 Falun, Sweden \\ ${ }^{2}$ Centre for Clinical Research in Dalarna, Nissers väg 3, 79182 Falun, Sweden \\ ${ }^{3}$ Division of Clinical Immunology, Department of Clinical and Experimental Medicine, Linköping University, \\ 58185 Linköping, Sweden \\ ${ }^{4}$ Division of Infectious Diseases, Department of Clinical and Experimental Medicine, Linköping University, 58185 Linköping, Sweden \\ ${ }^{5}$ Division of Pediatrics, Department of Clinical and Experimental Medicine, Linköping University, 58185 Linköping, Sweden \\ ${ }^{6}$ Department of Molecular Biology, Umeå University, 90187 Umeå, Sweden
}

Correspondence should be addressed to Barbro H. Skogman, barbro.hedinskogman@ltdalarna.se

Received 7 June 2011; Revised 25 August 2011; Accepted 29 August 2011

Academic Editor: Joanna Zajkowska

Copyright (C) 2012 Barbro H. Skogman et al. This is an open access article distributed under the Creative Commons Attribution License, which permits unrestricted use, distribution, and reproduction in any medium, provided the original work is properly cited.

Why some individuals develop clinical manifestations in Lyme borreliosis (LB) while others remain asymptomatic is largely unknown. Therefore, we wanted to investigate adaptive and innate immune responsiveness to Borrelia burgdorferi sensu lato in exposed Borrelia-antibody-positive asymptomatic children $(n=20)$, children with previous clinical LB $(n=24)$, and controls $(n=20)$. Blood samples were analyzed for Borrelia-specific interferon (IFN)- $\gamma$, interleukin (IL)-4, and IL-17 secretion by ELISPOT and Borrelia-induced IL-1 $\beta$, IL-6, IL-10, IL-12(p70), and tumor necrosis factor (TNF) secretion by Luminex. We found no significant differences in cytokine secretion between groups, but a tendency towards an increased spontaneous secretion of IL6 was found among children with previous clinical LB. In conclusion, the adaptive or innate immune responsiveness to Borrelia burgdorferi sensu lato was similar in Borrelia-exposed asymptomatic children and children with previous clinical LB. Thus, the immunological mechanisms of importance for eradicating the spirochete effectively without developing clinical manifestations of LB remain unknown.

\section{Introduction}

Lyme Borreliosis (LB), caused by the spirochete Borrelia (B.) burgdorferi, is the most common tick-borne infection in both Europe and the USA [1,2]. The infection may lead to a variety of symptoms by affecting different organs such as the skin, joints, heart muscle, or nervous system. The most common manifestation of $\mathrm{LB}$ is the migrating red skin lesion called erythema migrans (EM). LB in children follows a slightly different clinical course than in adults, and duration of symptoms is often shorter [3]. Children seem to have a better prognosis than adults and more seldom report persisting symptoms [4-6].
The cells of the innate immune system, constituting the first line of defense, recognize pathogen-associated molecular patterns (PAMPs) through pattern-recognition receptors (PRRs) like Toll-like receptors (TLRs) [7]. The spirochete $B$. burgdorferi contains a high proportion of lipoproteins that are mainly recognized by TLR2 [8]. Recognition of $B$. burgdorferi leads to the release of inflammatory mediators like interleukin (IL)-1 $\beta$, IL-6, IL-10, and IL-12, and tumor necrosis factor (TNF) from monocytes, macrophages, neutrophils, and DCs [9-12]. These cytokines are important for recruitment of other components of the innate host immune response but also for signaling with the adaptive immune 
system [12]. The adaptive immune system consists of $\mathrm{T}$ and $B$ lymphocytes and comprises the second line of defense to eliminate the spirochete. Activated T helper (Th) cells differentiate into Th1, Th2, Th17 or T-regulatory cells [13]. Th1 cells are important for immunity against intracellular pathogens, whereas Th2 cells are involved in immune responses against extracellular parasites [14]. Typical Th1 and Th2 effector cells are macrophages and mast cells, respectively. Th1 and Th2 are defined by their signature cytokines IFN- $\gamma$ and IL-4, which act antagonistically to counterbalance each other. Th17 cells, producing IL-17, are involved in the defense against fungi and some extracellular bacteria [15]. The immune response to B. burgdorferi involves both humoral and cell-mediated immune responses where both T-cell-independent and -dependent B cell responses are important during the adaptive immune response for killing the spirochetes [12].

Children and adults differ in the type of immune response they evoke when encountering B. burgdorferi.In adults, the immune response in LB is characterized by a strong Th1 responses with high numbers of Borrelia-specific IFN- $\gamma$-secreting cells and low levels of IL- $4[16,17]$, whereas children seem to have a more balanced immune response with elevated secretion of both IFN- $\gamma$ and IL-4 [18]. It has been hypothesized that the type of immune response evoked in the presence of the Borrelia spirochete may have significant effect on the clinical course and the outcome of the infection [19]. Persistent symptoms after LB were associated with a strong IFN- $\gamma$ response but lacked the subsequent upregulation of IL-4 [19]. Thus, it appears that a later prominent Th2 immune response is necessary to downregulate the initial strong IFN $-\gamma$ response in order to effectively terminate the infection and hinder unsatisfactory tissue damage. This could be one possible explanation why children usually experience a more benign course of the disease as they show both strong Th1 and Th2 immune responses [18].

The fact that some individuals may be exposed to $B$. burgdorferi s.l. without developing clinical symptoms is interesting from an immunological standpoint and could indicate a more effective immune response to the spirochete in these individuals. In adults, the term asymptomatic Borrelia infection is used for individuals who have been exposed to B. burgdorferi s.l. (i.e., with Borrelia IgG antibodies in serum) without known previous clinical LB [20]. These asymptomatic individuals have been found to have a higher secretion of the proinflammatory cytokines IL-12 and TNF than patients with clinical LB, suggesting an enhanced innate activity [21]. As for the adaptive immune responses, no differences have been found in Borrelia-specific IFN- $\gamma$ and IL4 secretion when comparing Borrelia exposed asymptomatic adults to patients with clinical LB [20]. To our knowledge, the innate and adaptive immune responses have not previously been studied in Borrelia exposed asymptomatic children.

The aim of this study was to investigate adaptive and innate immune responsiveness to Borrelia exposed asymptomatic children as compared to children with previous clinical LB to elucidate immunological mechanisms that might contribute to an effective eradication of the pathogen.

\section{Materials and Methods}

Children recruited to participate in this study were initially included in a larger prospective study, the ABIS (All Babies In Southeast Sweden) study, with the primary purpose of finding risk factors for immune-mediated diseases, mainly Type 1 diabetes $(n=17055)$. These children were followed until at 5 years of age at primary health care centers. All participating families completed a validated questionnaire, and venous or capillary blood samples were collected in conjunction with the 5-year followup. Two thousand children were randomly selected and screened for Borrelia IgG antibodies in serum [22]. Information concerning gender, geographic location, known tick bites, previous LB, and antibiotic treatment for LB was collected from the questionnaire.

Of these 2000 children, a total number of 64 children, geographically spread, were chosen to represent three major groups: Borrelia exposed asymptomatic children $(n=20)$, children with previous clinical LB $(n=24)$, and controls $(n=20)$ (Table 1). Borrelia exposed asymptomatic children were characterized by having Borrelia IgG antibodies in serum, but they reported no previous symptoms or treatment for LB in the questionnaire. Children with previous clinical LB reported previous treatment for LB, and some of them had Borrelia antibodies in serum (4/24). The control group reported no symptoms or previous treatment for LB and had no Borrelia antibodies in serum (Table 1). The control group and children with previous clinical LB were matched for gender and geographic location with Borrelia exposed asymptomatic children (Table 1). All 64 children were included for analysis with the ELISPOT assay but some later had to be excluded $(n=16)$ due to low responses in postive controls (see Data Handling). These excluded children did not differ statistically concerning gender (female $7 / 16$ versus $22 / 48$ ) or geographic location (rural living 7/16 versus 19/48) compared to included children $(n=48)$. Samples from 41 children were used for analysis with Luminex due to insufficiencies in cell samples (see Data Handling). The excluded children $(n=$ 23) did not differ statistically concerning gender (female $10 / 23$ versus 19/41) or geografic location (rural living $10 / 23$ versus $16 / 41)$ compared to included children $(n=$ 41). Informed consent has been given by all participating families, and the study was approved by the Regional Ethical Committee at the Faculty of Health Sciences, Linköping, Sweden (Dnr 03-547). All laboratory work in this study (i.e., not the collection and cryopreservation of cells) has been carried out by one person (S. Hellberg, one of the authors).

2.1. ELISA Antibody Test for B. burgdorferi s.l. A commercial enzyme-linked immunoassay (ELISA) kit, based on the Borrelia-specific protein Flagellin, was used (IDEA Borrelia burgdorferi IgG kit, DakoCytomation, Glostrup, Denmark 
TABLE 1: Subject characteristics at 5 years of age.

\begin{tabular}{lccc}
\hline & $\begin{array}{c}\text { Exposed asymptomatic } \\
(n=20)\end{array}$ & $\begin{array}{c}\text { Previous clinical LB } \\
(n=24)\end{array}$ & $\begin{array}{c}\text { Controls } \\
(n=20)\end{array}$ \\
\hline Gender $(\mathrm{f} / \mathrm{m})$ & $9 / 11$ & $11 / 13$ & $9 / 11$ \\
Rural living & 7 & 11 & 8 \\
Known tick bites & 20 & 24 & 0 \\
Previous clinical LB & & & 0 \\
$\quad$ EM & 0 & 22 & 0 \\
$\quad$ NB, facial nerve palsy & 0 & 5 & 0 \\
$\quad$ NB, meningitis & 0 & 1 & 0 \\
Antibiotic treatment for LB & 20 & 4 & 0 \\
Borrelia IgG antibodies in serum* & 0 & 4 & \\
\hline
\end{tabular}

Note. The data referred to in the table is given as numbers of children. Some of the children with previous clinical LB presented with several symptoms. n: number; f: female; m: male; LB: Lyme borreliosis; EM: Erythema migrans; NB: Neuroborreliosis; IgG: immunoglobulin G

$*$ Based on an ELISA (DAKO) kit for IgG antibodies for Borrelia-specific flagella antigen [24].

and Oxoid Limited, Hampshire, United Kingdom) [24], and cut-off for OD values was set according to the manufacturer's instructions.

2.2. Isolation, Cryopreservation, and Thawing of Peripheral Blood Mononuclear Cells (PBMCs). Blood samples were collected from the primary health centers, sent to the Division of Pediatrics, Linköping University, and prepared for isolation and cryopreservation as described in earlier studies [25]. When the time came for analyses, the samples were taken out of the nitrogen container and thawed in a $37^{\circ} \mathrm{C}$ water bath. Once thawed, the cell suspension was immediately transferred into a $15 \mathrm{~mL}$ polypropylene tube, and prewarmed $\left(37^{\circ} \mathrm{C}\right)$ tissue culture media (TCM) containing $10 \%$ heatinactivated fetal calf serum (FCS; Sigma Aldrich, Stockholm, Sweden) and Iscove's modification of Dulbecco's medium (GIBCO, Paisley, UK) supplemented with L-glutamine (Sigma Aldrich Sweden AB, Stockholm, Sweden) $292 \mathrm{mg} \mathrm{L}^{-1}$, MEM (minimum essential media) $100 \mathrm{X}$ nonessential amino acids $10 \mu \mathrm{g} \mathrm{mL}^{-1}$ (Invitrogen $\mathrm{AB}$, Paisley, UK), penicillin $50 \mathrm{IU} \mathrm{mL} L^{-1}$, streptomycin $50 \mu \mathrm{g} \mathrm{mL}^{-1}$ (BioWhittaker Europe, Essen, Germany), and $\mathrm{NaHCO}_{3} 3.024 \mathrm{~g} \mathrm{~L}^{-1}$ (Merck KGaA, Damstedt, Germany) were added dropwise along the wall of the tube to avoid osmotic shock in the cells. The cell suspension was centrifuged for 10 minutes at $400 \times \mathrm{g}$ at room temperature and the supernatant discarded. The cells were washed twice in TCM at $400 \times \mathrm{g}$ at RT for $10 \mathrm{~min}$. The cells were counted using a Bürker chamber in a phasecontrast microscope (Carl Zeiss AB, Stockholm, Sweden). The cell membrane integrity, that is, viability of the cells, was assessed with trypan blue exclusion and ranged from $83 \%$ to $99 \%$ with a median of $95 \%$. The concentration of cells was adjusted to $1 \times 10^{6} \mathrm{PBMC} \mathrm{mL}^{-1}$.

2.3. Preparation of the Borrelia Outer Surface Protein-Enriched Fraction (OF) Antigen. The cells, both in ELISPOT assay and in vitro stimulation for Luminex assay, were stimulated by $\mathrm{OF}$, consisting primarily of OspA and OspB from $B$. garinii strain Ip90. This antigen was chosen because it has previously been shown to be effective in differentiating between individuals with Borrelia infections and controls in ELISPOT assay for specific Borrelia IFN- $\gamma$ and IL-4 secretion $[17,23]$. The antigen was prepared as described in earlier reports [26, 27], and the optimal concentration of the Borrelia OF antigen was determined through testing of different antigen concentrations $\left(54,18,6\right.$, and $2 \mu \mathrm{g} \mathrm{m}^{-1}$ ) on an adult patient sample with confirmed neuroborreliosis (NB) by ELISPOT analysis. The final optimal concentration of Borrelia OF antigen was therefore $6 \mu \mathrm{g} / \mathrm{mL}^{-1}$ in the ELISPOT assay. In the Luminex assay, a concentration of $12 \mu \mathrm{g} / \mathrm{mL}$ was used.

2.4. ELISPOT Analysis for IFN- $\gamma, I L-4$, and IL-17. To determine the T-cell response to B. burgdorferi s.l., an ELISPOT assay was used to assess the number of Borrelia-specific IFN$\gamma$-, IL-4- and IL-17-secreting cells. The ELISPOT analyses, originally described by Czerkinsky et al. [28], were performed according to the instructions provided by the manufacturer (Mabtech AB, Nacka, Sweden) and as described in detail in earlier studies $[17,23]$. Tetanus toxoid (TT; Swedish Institute for Infectious Disease Control, Stockholm, Sweden) and phytohemagglutinin A (PHA; Sigma-Aldrich $\mathrm{AB}$, Stockholm, Sweden) were used as positive controls, representing recall antigen and polyclonal stimulation, respectively, at a final concentration of $5 \mathrm{LF}$ units $\mathrm{mL}^{-1}$ for TT and $20 \mu \mathrm{g} \mathrm{mL}^{-1}$ for PHA. A peptide pool consisting of 32 peptides derived from the human Cytomegalovirus, Epstein-Barr virus, and Influenza virus (CEF; Mabtech $A B$, Nacka, Sweden) was also included as an additional positive control for IFN- $\gamma$ responses and used at a final concentration of $2 \mu \mathrm{g} \mathrm{mL}^{-1}$. As for negative controls, wells with TCM were used without cells. All samples (except wells containing only PHA or cell medium alone) were assayed in triplicate although some samples could only be assayed in duplicate due to low cell count. The spots were counted manually by dissection microscope and by semiautomatic AID EliSpot Reader system/HR version 3.2.3 (AID autoimmune diagnostics GmbH, Strassberg, Germany) in a blinded manner on one single occasion by the same 
person (S. Hellberg, one of the authors). Each spot counted represented one cytokine-producing cell.

2.5. Luminex Analysis for $I L-1 \beta, I L-6, I L-10, I L-12(p 70)$, and TNF. Half a million PMBCs diluted in $0.5 \mathrm{~mL}$ TCM supplemented with 10\% FCS (Sigma Aldrich, Stockholm, Sweden) were cultured together with OF from B. garinii and LPS (Sigma-Aldrich AB, Stockholm, Sweden) from Salmonella typhimurium at final concentrations of $12 \mu \mathrm{g} \mathrm{mL}-1,1 \mathrm{ng}$ $\mathrm{mL}^{-1}$, and $100 \mathrm{ng} \mathrm{mL}^{-1}$, respectively, or without antigen, at $37^{\circ} \mathrm{C}, 5 \% \mathrm{CO}_{2}$, for 24 hours. After 24 hours, the cells were centrifuged $(400 \times \mathrm{g}$ at RT), and the supernatants were collected and frozen at $-70^{\circ} \mathrm{C}$ until used for the Luminex analysis of cytokines in the samples. The levels of cytokines IL-1 $\beta$, IL-6, IL-10, IL-12(p70), and TNF were measured in the PBMC supernatants by a Bio-Plex Pro Human Cytokine Panel Kit (Bio-Rad Laboratories, Calif, USA). All assays were carried out in accordance with the instructions provided by the manufacturer. The plates were then analyzed using Luminex 200 (Invitrogen, Merelbeke, Belgium). The analysis condition was set to a minimum of 100 beads per region. The raw data, median fluorescent intensity (MFI), was analyzed using xPONENT 3.1 (Luminex Corporation, Austin, Tex, USA). The quantifiable ranges for the standard curves were for IL-1 $\beta$ 1.91-1959.31 $\mathrm{pg} \mathrm{mL}^{-1}$, IL-6 1.54-25171 $\mathrm{pg} \mathrm{mL}^{-1}$, IL-10 1.48-6076 pg mL ${ }^{-1}$, TNF 4.75-19438.75 $\mathrm{pg} \mathrm{mL}^{-1}$, and for IL-12(p70) 2.19-8988 $\mathrm{pg} \mathrm{mL}^{-1}$. Values below the lowest detection limit of the standard curve were assigned half the value of the detection limit, and values above highest detection limit were assigned double the value of the detection limit.

2.6. Data Handling. Regarding the ELISPOT assay, the median of the triplicates or duplicates was used for the analysis of cytokine-secreting cells. The method for determining Borrelia-specific secretion in this study has previously been implemented in other studies [18-20] and is based on both the unstimulated, spontaneous secretion and the antigenstimulated secretion of the cells. The Borrelia-specific secretion was determined by subtracting the number of spots from the wells with cell suspension and medium (i.e., the spontaneous secretion) from the number of spots in the OF-antigen-stimulated wells (i.e., Borrelia-stimulated secretion) [20]. Both the Borrelia-specific secretion and the spontaneous secretion are interesting to show to give a full picture of immune responses, and both are therefore reported in Results, Discussion, and Figures.

All 64 samples were investigated by the ELISPOT assay. However, as there were low responses in some of the positive controls, defined criteria were used to assure that cells had the ability to respond. These criteria were mainly based on the PHA responses for IFN- $\gamma$. Samples with a high PHA response for IFN- $\gamma$ (over 300 spots) were all included $(n=27)$. Samples with PHA response of 200-300 spots were included if they also had an apparent antigen-induced response for TT and CEF (for more than one cytokine) or a strong PHA response for both IL-4 and IL-17 $(n=19)$. Based on previous experience, samples with a PHA response for IFN- $\gamma$ showing less than 200 spots were excluded $(n=16)$ with the exception of two samples showing strong antigeninduced responses for both TT and CEF (for more than two cytokines) and strong PHA responses for both IL-4 and IL$17(n=2)$. All these considerations were blinded from the belonging to groups and the Borrelia-stimulated secretion.

With regard to the Luminex assay, 41 samples were available and all were analyzed. The ability of the cells to respond to stimuli was assessed by the ratio between the samples stimulated with LPS and the spontaneous secretion. Samples were included if they had an LPS/spontaneous secretion ratio of five or more for any of the analyzed cytokines. Based on these criteria, all samples were valid and could be included for analysis $(n=41)$. The Borrelia induced secretion was obtained by subtracting spontaneous secretion from the samples stimulated with Borrelia OF antigen as previously implemented for the ELISPOT assay.

2.7. Statistical Analysis. Statistical Products and Service Solutions (SPSS), version 17.0 for Windows was used for the statistical analysis. The Kruskal-Wallis test was used as a pretest for comparison of the immunological parameters between the groups. Mann-Whitney $U$ test was used as a post hoc test when the $P$ value for Kruskal-Wallis test was $P<$ 0.08. For Mann-Whitney, a level of $P<0.05$ was considered statistically significant. Since the cytokines analyzed in this study were viewed as part of a pattern and not as separate events, no corrections for multiple comparisons were made.

\section{Results}

3.1. Number of IFN- $\gamma$-, IL-4- and IL-17- Secreting Cells Measured by ELISPOT. All groups displayed a predominance of Borrelia-specific IFN- $\gamma$-secreting cells as compared to IL4- and IL-17-secreting cells. However, no significant differences were found between Borrelia exposed asymptomatic children and children with previous clinical LB regarding the number of Borrelia-specific IFN- $\gamma$-, IL-4-, and IL-17secreting cells (Figure 1). Moreover, no significant difference could be found in comparison to the control group for any of the analyzed cytokines. Some of the individuals in all three groups had negative values for the Borrelia-specific secretion, that is, the spontaneous secretion was higher than the OFstimulated secretion (Figure 1).

When analyzing the number of spontaneous secreting cells, indicating the nonstimulated background activity of cytokine secretion, no significant differences in IFN- $\gamma$, IL4 , or IL-17 was found between any of the groups (Figure 2). A ratio between the numbers of IL-4- and IFN- $\gamma$ - spontaneously secreting cells, assessing the Th2/Th1 balance, did not show any significant differences across groups (data not shown). PHA-induced secretion, indicating the ability of the cells to respond to mitogenic stimulation, elicited a stronger response for IFN- $\gamma$ than for IL-4 and IL-17, but no differences were found between groups (data not shown).

3.2. IL-1 $\beta, I L-6, I L-10, I L-12(p 70)$, and TNF Secretion Measured by Luminex. The Borrelia induced secretion of IL-1 $\beta$, IL-6, IL-10, and TNF were readily detectable with the 
TABLE 2: The OF induced cytokine secretion in PBMCs by Luminex.

\begin{tabular}{lccc}
\hline Cytokine & Exposed asymptomatic & Previous clinical LB & Controls \\
\hline IL-1 $\beta$ & $309(183-846)$ & $341(132-1223)$ & $340(162-800)$ \\
IL-6 & $6805(3528-12749)$ & $7304(3281-50303)$ & $7335(2578-12324)$ \\
IL-10 & $130(38-442)$ & $129(42-572)$ & $123(37-684)$ \\
IL-12(p70) & $1(0-1)$ & $1(0-1)$ & $1(0-1)$ \\
TNF & $676(241-2311)$ & $561(209-1724)$ & $628(226-1373)$ \\
\hline
\end{tabular}

NOTE. The data referred to in the table is given as median values in $\mathrm{pg} / \mathrm{mL}$ (range in parenthesis).

OF: outer surface protein enriched fraction; PBMCs: peripheral blood mononuclear cells; LB: Lyme Borreliosis; IL = interleukin; TNF: tumour necrosis factor.

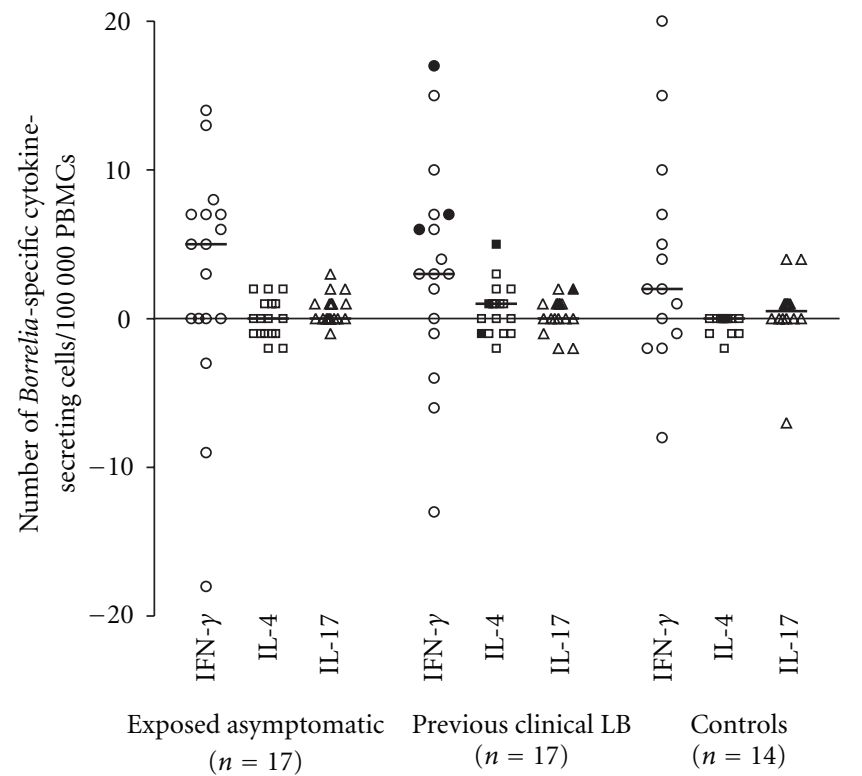

Figure 1: The number of Borrelia-specific IFN- $\gamma$-secreting cells (open circle), IL-4-secreting cells (open square) and IL-17-secreting cells (open triangle), per 100000 PBMCs as measured by ELISPOT in different groups. The filled circles, squares, and triangles represent children in the previous clinical LB group with Borrelia seropositivity. The Borrelia-specific secretions are net values obtained after subtracting the number of spontaneous cytokinesecreting cells from the number of outer surface protein fraction (OF-) antigen-specific cytokine-secreting cells. The median values are noted as lines in the figure. No statistically significant differences were found between groups.

Luminex assay, whereas the Borrelia induced secretion of IL12(p70) was undetectable in all samples (Table 2). Moreover, the IL-12(p70) levels were also below the detection limit also in 32 of the 40 samples stimulated with LPS and did not exceeded $8 \mathrm{pg} / \mathrm{mL}$ in the remaining 8 samples. Thus, levels of IL-12(p70) were not considered interpretable. Of the remaining cytokines, IL- 6 was present in the overall highest concentration in all groups and IL-10 in the lowest concentration in all groups (Table 2).

As for the Borrelia induced secretion, no significant differences were found between Borrelia exposed asymptomatic children, children with previous clinical LB, and controls with regard to IL-1 $\beta$, IL-6, IL-10, and TNF (Figure 3). The spontaneous secretion did not differ significantly between

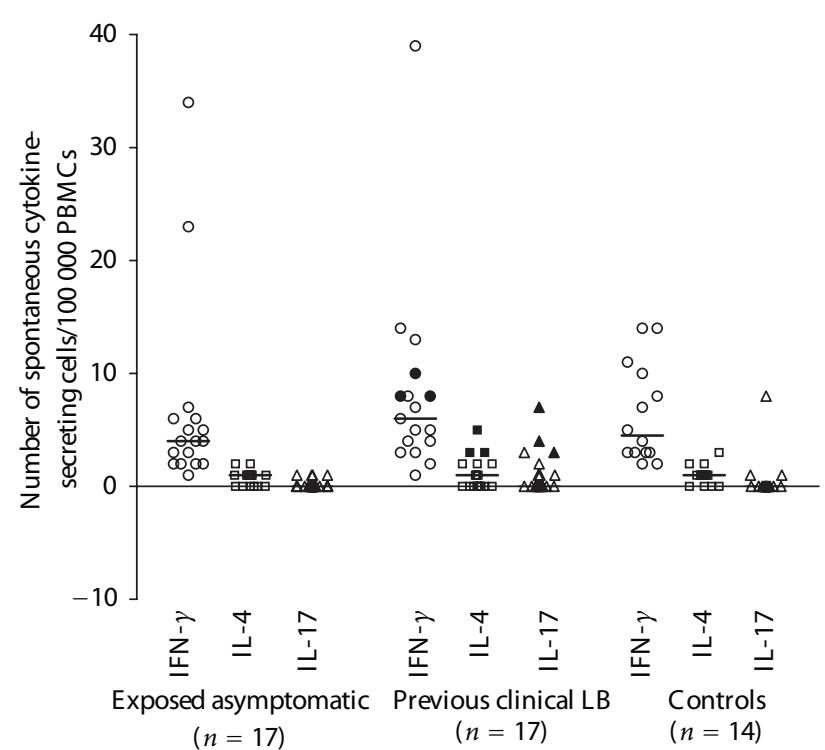

FIGURE 2: The number of spontaneously IFN- $\gamma$-secreting cells (open circle), IL-4-secreting cells (open square), and IL-17secreting cells (open triangle) per 100000 PBMCs as measured by ELISPOT in different groups. The filled circles, squares, and triangles represent the children in the previous clinical LB group with Borrelia seropositivity. The median values are noted as lines in the figure. No statistically significant differences were found between groups.

groups for any of the cytokines although there was a tendency $(P=0.057)$ towards an increased spontaneous secretion of IL-6 in children with previous clinical LB as compared to Borrelia exposed asymptomatic children (Figure 4). The ratio between LPS-stimulated secretion and the spontaneous secretion, indicating the ability of the cells to respond to a TLR4-agonistic stimulus, was (median values with range in parenthesis): 301(9-2051) for IL-1 $\beta$; 314(8-4262) for IL6 ; 134(17-450) for IL-10 and 66(7-652) for TNF (data not shown).

\section{Discussion}

In this study, we have investigated the Borrelia-specific (adaptive) and the Borrelia induced (innate) immune responses in Borrelia exposed asymptomatic children and children with 

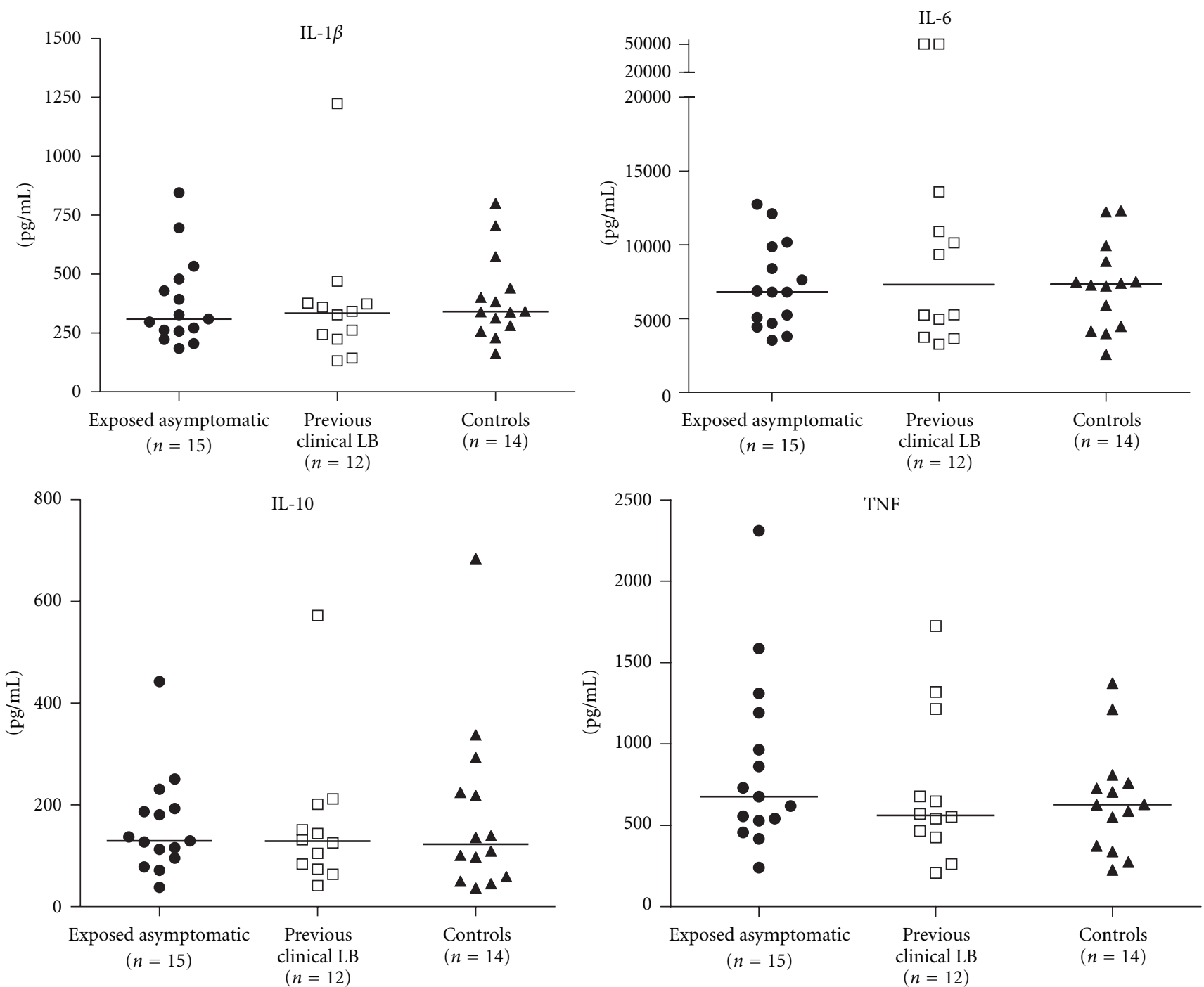

Figure 3: The Borrelia-induced secretion of IL-1 $\beta$, IL-6, IL-10, and TNF in PBMC supernatants from Borrelia exposed asymptomatic children (filled circle), children with previous clinical LB (open square), and controls (filled triangle) as measured by Luminex. The Borrelia induced secretions are net values obtained after subtracting the level of spontaneous cytokine secretion from the level of outer surface protein-fraction (OF-) stimulated cytokine secretion. The median values are noted as lines in the figure. No statistically significant differences were found between groups.

previous clinical LB. Our aim was to better understand immunological mechanisms that could explain why some individuals develop clinical manifestations of LB and others do not. Interestingly, we found no differences in the number of Borrelia-specific IFN- $y$-, IL-4-, and IL-17-secreting cells when comparing Borrelia exposed asymptomatic children, children with previous clinical LB, and controls. This lack of Borrelia-specific (adaptive) immune responsiveness is congruent with earlier studies on Borrelia exposed asymptomatic adults where no differences in the number of Borrelia-specific IFN- $\gamma$ - and IL-4-secreting cells were found compared to patients with clinical LB [20]. Our negative findings are further supported by Jarefors et al. [29], who found no differences in IFN- $\gamma$ secretion between asymptomatic adults and patients with previous clinical LB [29]. As for the innate immune responses, no significant differences in the Borrelia induced cytokine IL-1 $\beta$, IL-6, IL-10, or TNF secretion between groups were found in our study, whereas in a previous study by Sjöwall et al. [21], an increased number of Borrelia-induced TNF-secreting DCs were found in asymptomatic adults as compared to patients with a history of NB [21].

We are well aware of the fact that the number of patients in each group is rather low; but with well-characterized patient groups, a proper design of the study and nonparametric statistic calculations, the results should be reliable. Furthermore, the fact that the number of Borrelia-specific IFN- $\gamma$ - and IL-4-secreting cells was generally lower as compared to earlier studies $[17,18,20]$ led us to consider that the responsiveness of the cells to stimuli might have been reduced due to the quality of the cells after freezing (previous studies were performed on freshly isolated cells). 

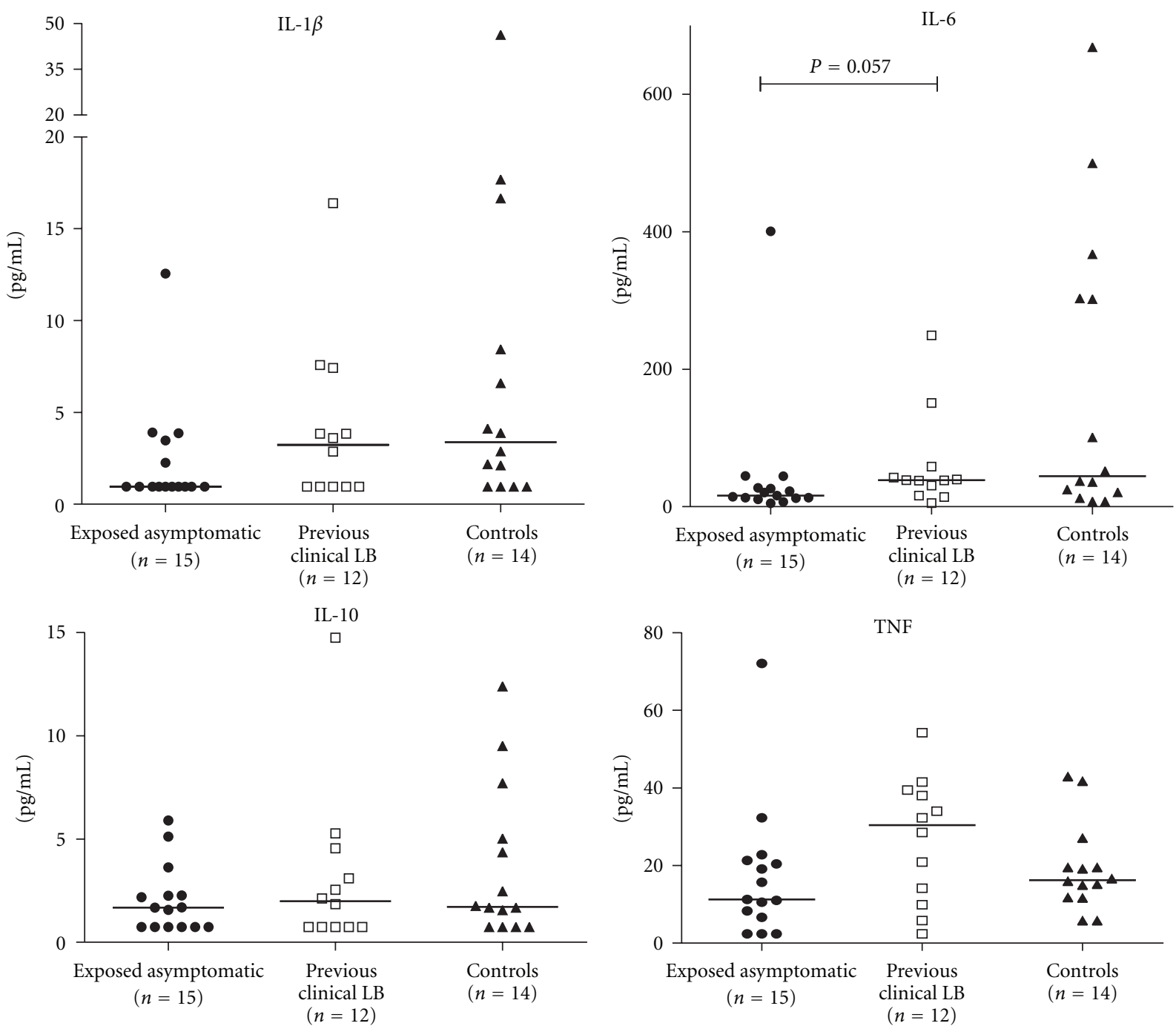

FIgURE 4: The spontaneous secretion of IL-1 $\beta$, IL-6, IL-10, and TNF in PBMC supernatants from Borrelia exposed asymptomatic children (filled circle), children with previous clinical LB (open square), and controls (filled triangle) as measured by Luminex. The median values are noted as lines in the figure. There was a tendency to higher IL-6 in children with previous clinical LB compared to Borrelia exposed asymptomatic children, otherwise no statistically significant differences were found between groups.

However, the response to PHA was similar to that obtained from another study on LB in adults [17], and additionally the response to LPS was substantially higher than the spontaneous secretion which, considered together, confirms the ability of the cells to respond to stimuli. The use of cryopreserved PBMCs in this study was necessary due to the design of the study, as it would have been impossible to test cellular immune responses in freshly isolated PBMCs from thousands of unselected children. Earlier reports on cryopreservation effects on cytokine secretion by ELISPOT showed a general decrease in the IL- 4 secretion in cryopreserved cells as compared to fresh cells both in spontaneous and allergen-induced secretion, whereas IFN- $\gamma$ secretion was less affected [30]. Thus, to some extent, the cryopreservation could explain the low levels of IL-4-secreting cells found in this study as compared to earlier studies with freshly prepared PBMCs from children (1-17 years old) [18]. It is also important to note that blood samples from children were taken postinfection and not during the actual infection, in accordance with the design of the study.

The young age of the children has also to be taken into consideration. The ability to respond with IFN- $\gamma$ is impaired in neonates [31] and develops during childhood [32, 33]. The children in our study were all 5 years of age, and thus one would expect full capacity to respond with IFN- $\gamma$ to stimuli $[34,35]$. A similar capacity was found when comparing the PHA-induced IFN- $\gamma$ secretion in our present study to the PHA-induced IFN- $\gamma$ secretion in adults [17].

Whether or not children with previous clinical LB are "truly" Borrelia exposed, patients may be a matter for discussion. Data in our present study is based on selfreported information that may have weaknesses. However, most patients did report a previous EM, which is a clear clinical diagnosis, and although self-reported, the diagnosis 
was stated to be set by a physician and treated with antibiotics. Very few of the children with previous clinical LB were Borrelia seropositive $(n=4$, Table 1$)$, but this was expected since EM is a clinical diagnosis set by a physician, the sensitivity of the test in EM is low and antibody levels may be reduced after antibiotic treatment [36]. Previous antibiotic treatment in this group could theoretically also have influenced immune responses. Moreover, one must remember that our study mainly evaluates children with previous EM (only 5 children with facial palsy and 1 with meningitis), thus conclusions on immune responses in disseminated LB could not be drawn from our data.

Furthermore, whether or not Borrelia exposed asymptomatic children are "truly" Borrelia exposed or falsely seropositive may also be a matter for discussion. We have not carried out any confirmatory test since the specificity of the test is high [37], and in earlier studies, a Borrelia-specific T-cell response in PBMCs was noted in Borrelia exposed asymptomatic adults, certifying a true exposure [38]. Thus, we believe that false seropositive specimens should not be a problem in our material.

We found a tendency towards higher levels of spontaneous secretion of IL-6 (by Luminex) in children with previous clinical LB (Figure 4). IL-6 is a pleiotropic cytokine that mainly mediates proinflammatory effects, and it induces secretion of IL-17 from naïve T cells [39] and may therefore, together with IL-17, be involved in pathogenesis of LB. Recently, it was suggested that IL-17 might contribute to the pathogenesis in Lyme arthritis and in NB [40, 41]. Thus, one could speculate that IL-6 together with IL-17 may be involved in inflammatory mechanisms contributing to clinical manifestations of LB. However, in the present study, we found only a tendency of elevated spontaneous secretion of IL- 6 but no Borrelia induced IL- 6 or IL- 17 secretion. This could possibly be explained by the fact that we have analyzed PBMCs after inflammation and not specimen from immune privileged sites during active inflammation. Thus, the role of IL-6 together with IL-17 is certainly interesting regarding the pathogenesis in LB but needs further investigation.

Finally, why some individuals develop a clinical disease upon encountering B. burgdorferi s.l. and some do not remains unclear. Whether or not specific properties of the host's immune system are of importance is still not understood, and, admittedly, there might be other important aspects. For example, the Borrelia genospecies infecting the human might play a substantial role in the different clinical outcomes observed in LB. It is well established that the different genospecies of B. burgdorferi s.l. can cause different clinical manifestations, and it is also well known that different genospecies have different abilities in escaping the complement system, thereby avoiding elimination [42]. These aspects, as well as the individual genetic predisposition might be crucial factors in understanding mechanisms in the spirochete-host interaction, and future studies are warranted in these fields.

In conclusion, our results show no differences in adaptive or innate immune responsiveness to B. burgdorferi s.l. when comparing Borrelia exposed asymptomatic children and children with previous clinical LB. Thus, immunological mechanisms of importance for eradicating the spirochete effectively without developing clinical manifestations of LB remain unknown.

\section{List of Abbreviations}

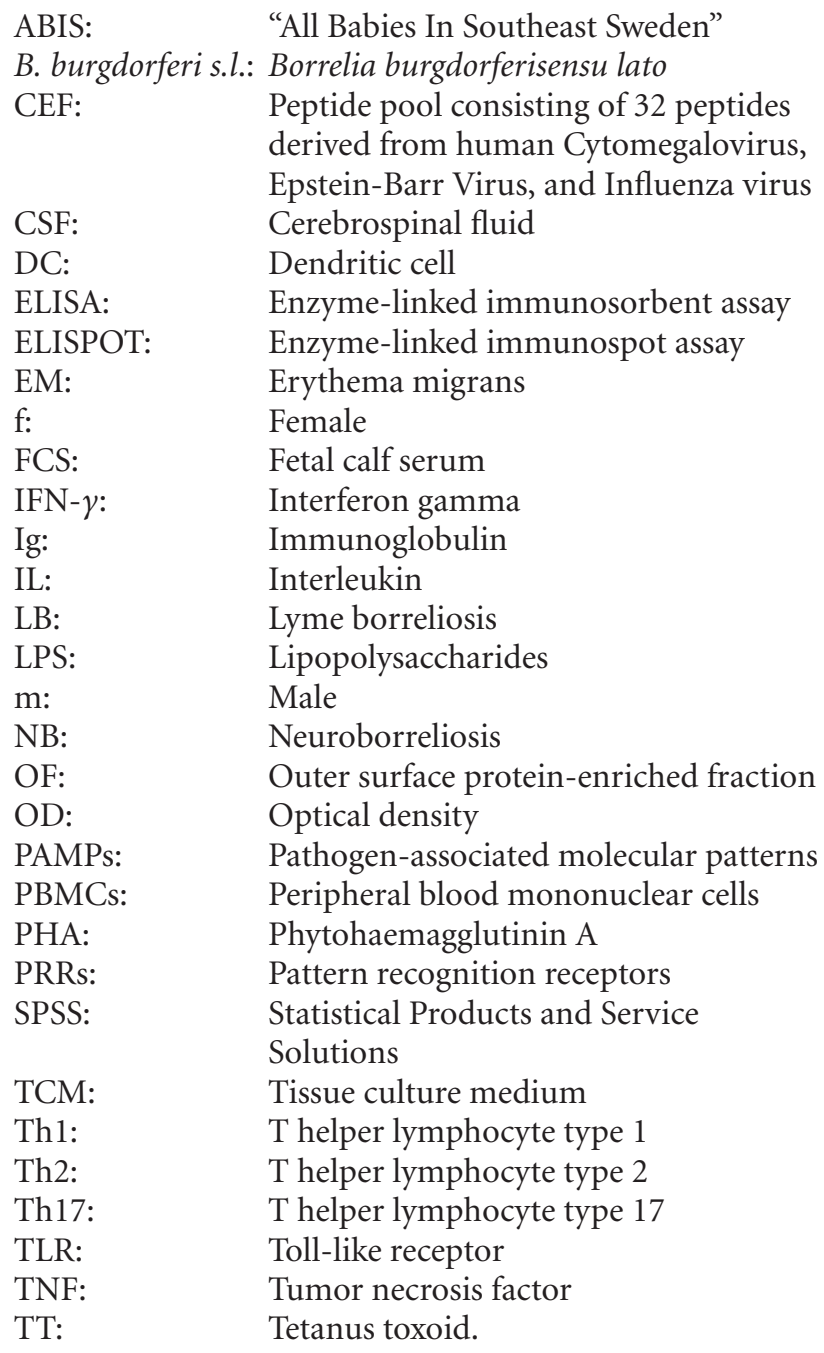

\section{Conflict of Interest}

No conflict of interests is stated by the authors.

\section{Acknowledgment}

The authors are most grateful to all participating children/parents. They also wish to thank the staff at the Child Health Services in the region as well as the excellent research nurses and the technicians connected to the ABIS study, in particular Ingela Johansson and Gosia Smolinska. Also special thanks are due to Mari-Anne Åkeson and Petra Cassel for excellent expertise on ELISPOT and Luminex analysis, respectively. In addition, they are most grateful to the staff at the Department of Molecular Biology, Umeå University for supplying the Borrelia OF antigen. The study was supported by grants from the Research Council in Southeast Sweden 
(FORSS), the County Council in Östergötland, the Swedish Child Diabetes Foundation, the Juvenile Diabetes Research Foundations, the Holmia Foundation and the Center of Clinical Research in Dalarna (CKF).

\section{References}

[1] G. Stanek and F. Strle, "Lyme borreliosis," Lancet, vol. 362, no. 9396, pp. 1639-1647, 2003.

[2] A. C. Steere, "Lyme borreliosis in 2005, 30 years after initial observations in lyme connecticut," Wiener Klinische Wochenschrift, vol. 118, no. 21-22, pp. 625-633, 2006.

[3] H. J. Christen, F. Hanefeld, H. Eiffert, and R. Thomsen, "Epidemiology and clinical manifestations of Lyme borreliosis in childhood. A prospective multicentre study with special regard to neuroborreliosis," Acta Paediatrica, Supplement, vol. 82 , no. 386, pp. 1-75, 1993.

[4] J. Berglund, L. Stjernberg, K. Ornstein, K. Tykesson-Joelsson, and H. Walter, "5-y follow-up study of patients with neuroborreliosis," Scandinavian Journal of Infectious Diseases, vol. 34, no. 6, pp. 421-425, 2002.

[5] M. Vázquez, S. S. Sparrow, and E. D. Shapiro, "Long-term neuropsychologic and health outcomes of children with facial nerve palsy attributable to lyme disease," Pediatrics, vol. 112, no. 2, pp. e93-e97, 2003.

[6] B. H. Skogman, S. Croner, M. Nordwall, M. Eknefelt, J. Ernerudh, and P. Forsberg, "Lyme neuroborreliosis in children: a prospective study of clinical features, prognosis, and outcome," Pediatric Infectious Disease Journal, vol. 27, no. 12, pp. 1089-1094, 2008.

[7] R. Medzhitov and C. Janeway Jr., "The Toll receptor family and microbial recognition," Trends in Microbiology, vol. 8, no. 10, pp. 452-456, 2000.

[8] M. Hirschfeld, G. J. Kirschning, R. Schwandner et al., "Cutting edge: inflammatory signaling by Borrelia burgdorferi lipoproteins is mediated by toll-like receptor 2," Journal of Immunology, vol. 163, no. 5, pp. 2382-2386, 1999.

[9] J. Suhonen, J. Komi, J. Soukka, O. Lassila, and M. K. Viljanen, "Interaction between Borrelia burgdorferi and immature human dendritic cells," Scandinavian Journal of Immunology, vol. 58, no. 1, pp. 67-75, 2003.

[10] V. A. Dennis, S. Dixit, S. M. O’Brien, X. Alvarez, B. Pahar, and M. T. Philipp, "Live Borrelia burgdorferi spirochetes elicit inflammatory mediators from human monocytes via the tolllike receptor signaling pathway," Infection and Immunity, vol. 77, no. 3, pp. 1238-1245, 2009.

[11] A. C. Steere, J. Coburn, and L. Glickstein, "The emergence of Lyme disease," Journal of Clinical Investigation, vol. 113, no. 8, pp. 1093-1101, 2004.

[12] M. D. McKisic and S. W. Barthold, "T-cell-independent responses to Borrelia burgdorferi are critical for protective immunity and resolution of lyme disease," Infection and Immunity, vol. 68, no. 9, pp. 5190-5197, 2000.

[13] J. Zhu and W. E. Paul, "CD4 T cells: fates, functions, and faults," Blood, vol. 112, no. 5, pp. 1557-1569, 2008.

[14] T. R. Mosmann and S. Sad, "The expanding universe of T-cell subsets: Th1, Th2 and more," Immunology Today, vol. 17, no. 3, pp. 138-146, 1996.

[15] A. Peck and E. D. Mellins, "Precarious balance: Th17 cells in host defense," Infection and Immunity, vol. 78, no. 1, pp. 32$38,2010$.

[16] J. Oksi, J. Savolainen, J. Pène, J. Bousquet, P. Laippala, and M. K. Viljanen, "Decreased interleukin-4 and increased gamma interferon production by peripheral blood mononuclear cells of patients with Lyme borreliosis," Infection and Immunity, vol. 64, no. 9, pp. 3620-3623, 1996.

[17] C. Ekerfelt, J. Ernerudh, J. Bunikis et al., "Compartmentalization of antigen specific cytokine responses to the central nervous system in CNS borreliosis: secretion of IFN$\gamma$ predominates over IL-4 secretion in response to outer surface proteins of lyme disease Borrelia spirochetes," Journal of Neuroimmunology, vol. 79, no. 2, pp. 155-162, 1997.

[18] M. Widhe, B. H. Skogman, S. Jarefors et al., "Up-regulation of Borrelia-specific IL-4- and IFN- $\gamma$-secreting cells in cerebrospinal fluid from children with Lyme neuroborreliosis," International Immunology, vol. 17, no. 10, pp. 1283-1291, 2005.

[19] M. Widhe, S. Jarefors, C. Ekerfelt et al., "Borrelia-specific interferon- $\gamma$ and interleukin-4 secretion in cerebrospinal fluid and blood during lyme borreliosis in humans: association with clinical outcome," Journal of Infectious Diseases, vol. 189, no. 10, pp. 1881-1891, 2004.

[20] C. Ekerfelt, P. Forsberg, M. Svenvik, M. Roberg, S. Bergström, and J. Ernerudh, "Asymptomatic Borrelia-seropositive individuals display the same incidence of Borrelia-specific interferon-gamma (IFN- $\gamma$ )-secreting cells in blood as patients with clinical Borrelia infection," Clinical and Experimental Immunology, vol. 115, no. 3, pp. 498-502, 1999.

[21] J. Sjöwall, A. Carlsson, O. Vaarala et al., "Innate immune responses in Lyme borreliosis: enhanced tumour necrosis factor- $\alpha$ and interleukin-12 in asymptomatic individuals in response to live spirochetes," Clinical and Experimental Immunology, vol. 141, no. 1, pp. 89-98, 2005.

[22] B. H. Skogman, C. Ekerfelt, J. Ludvigsson, and P. Forsberg, "Seroprevalence of Borrelia IgG antibodies among young Swedish children in relation to reported tick bites, symptoms and previous treatment for Lyme borreliosis: a populationbased survey," Archives of Disease in Childhood, vol. 95, no. 12, pp. 1013-1016, 2010.

[23] P. Forsberg, J. Ernerudh, C. Ekerfelt, M. Roberg, M. Vrethem, and S. Bergstrom, "The outer surface proteins of Lyme disease Borrelia spirochetes stimulate $\mathrm{T}$ cells to secrete interferongamma (IFN- $\gamma$ ): diagnostic and pathogenic implications," Clinical and Experimental Immunology, vol. 101, no. 3, pp. 453-460, 1995.

[24] K. Hansen and E. Asbrink, "Serodiagnosis of erythema migrans and acrodermatitis chronica atrophicans by the Borrelia burgdorferi flagellum enzyme-linked immunosorbent assay," Journal of Clinical Microbiology, vol. 27, no. 3, pp. 545$551,1989$.

[25] S. Axelsson, M. Faresjö, M. Hedman, J. Ludvigsson, and R. Casas, "Cryopreserved peripheral blood mononuclear cells are suitable for the assessment of immunological markers in type 1 diabetic children," Cryobiology, vol. 57, no. 3, pp. 201-208, 2008.

[26] S. Bergstrom, A. Sjostedt, L. Dotevall et al., "Diagnosis of Lyme borreliosis by an enzyme immunoassay detecting immunoglobulin G reactive to purified Borrelia burgdorferi cell components," European Journal of Clinical Microbiology and Infectious Diseases, vol. 10, no. 5, pp. 422-427, 1991.

[27] L. A. Magnarelli, J. F. Anderson, and A. G. Barbour, "Enzymelinked immunosorbent assays for lyme disease: reactivity of subunits of Borrelia burgdorferi," Journal of Infectious Diseases, vol. 159, no. 1, pp. 43-49, 1989.

[28] C. Czerkinsky, G. Andersson, H. P. Ekre, L. A. Nilsson, L. Klareskog, and O. Ouchterlony, "Reverse ELISPOT assay for clonal analysis of cytokine production. I. Enumeration of 
gamma-interferon-secretion cells," Journal of Immunological Methods, vol. 110, no. 1, pp. 29-36, 1988.

[29] S. Jarefors, C. K. Janefjord, P. Forsberg, M. C. Jenmalm, and C. Ekerfelt, "Decreased up-regulation of the interleukin-12R $\beta 2$ chain and interferon- $\gamma$ secretion and increased number of forkhead box P3-expressing cells in patients with a history of chronic Lyme borreliosis compared with asymptomatic Borrelia-exposed individuals," Clinical and Experimental Immunology, vol. 147, no. 1, pp. 18-27, 2007.

[30] M. Kvarnström, M. C. Jenmalm, and C. Ekerfelt, "Effect of cryopreservation on expression of Th1 and Th2 cytokines in blood mononuclear cells from patients with different cytokine profiles, analysed with three common assays: an overall decrease of interleukin-4," Cryobiology, vol. 49, no. 2, pp. 157-168, 2004.

[31] Y. J. Bryson, H. S. Winter, and S. E. Gard, "Deficiency of immune interferon production by leukocytes of normal newborns," Cellular Immunology, vol. 55, no. 1, pp. 191-200, 1980.

[32] P. G. Holt and C. A. Jones, "The development of the immune system during pregnancy and early life," Allergy, vol. 55, no. 8, pp. 688-697, 2000.

[33] M. F. Böttcher, M. C. Jenmalm, and B. Björkstén, "Immune responses to birch in young children during their first 7 years of life," Clinical and Experimental Allergy, vol. 32, no. 12, pp. 1690-1698, 2002.

[34] F. Hoffmann, M. H. Albert, S. Arenz et al., "Intracellular Tcell cytokine levels are age-dependent in healthy children and adults," European Cytokine Network, vol. 16, no. 4, pp. 283$288,2005$.

[35] V. Wiegering, M. Eyrich, C. Wunder, H. Günther, P. G. Schlegel, and B. Winkler, "Age-related changes in intracellular cytokine expression in healthy children," European Cytokine Network, vol. 20, no. 2, pp. 75-80, 2009.

[36] S. Hammers-Berggren, A. M. Lebech, M. Karlsson, B. S. Ngsson, K. Hansen, and G. Stiernstedt, "Serological followup after treatment of patients with erythema migrans and neuroborreliosis," Journal of Clinical Microbiology, vol. 32, no. 6, pp. 1519-1525, 1994.

[37] A. Smismans, V. J. Goossens, E. Nulens, and C. A. Bruggeman, "Comparison of five different immunoassays for the detection of Borrelia burgdorferi Igm and IgG antibodies," Clinical Microbiology and Infection, vol. 12, no. 7, pp. 648-655, 2006.

[38] C. Ekerfelt, C. Masreliez, M. Svenvik, J. Ernerudh, M. Roberg, and P. Forsberg, "Antibodies and T-cell reactivity to Borrelia burgdorferi in an asymptomatic population: a study of healthy blood donors in an Inland town district in the South-East of Sweden," Scandinavian Journal of Infectious Diseases, vol. 33, no. 11 , pp. 806-808, 2001.

[39] F. Sallusto and A. Lanzavecchia, "Human Th17 cells in infection and autoimmunity," Microbes and Infection, vol. 11, no. 5, pp. 620-624, 2009.

[40] A. J. Henningsson, I. Tjernberg, B. E. Malmvall, P. Forsberg, and J. Ernerudh, "Indications of Th1 and Th17 responses in cerebrospinal fluid from patients with Lyme neuroborreliosis: a large retrospective study," Journal of Neuroinflammation, vol. 8,2011 .

[41] M. Nordberg, P. Forsberg, A. Johansson et al., "Cytotoxic mechanisms may play a role in the local immune response in the central nervous system in neuroborreliosis," Journal of Neuroimmunology, vol. 232, no. 1, pp. 186-193, 2011.

[42] A. Alitalo, T. Meri, H. Lankinen et al., "Complement inhibitor factor $\mathrm{H}$ binding to lyme disease spirochetes is mediated by inducible expression of multiple plasmid-encoded outer surface protein E paralogs," Journal of Immunology, vol. 169, no. 7, pp. 3847-3853, 2002. 


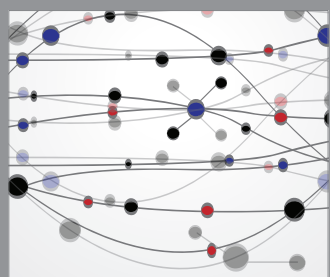

The Scientific World Journal
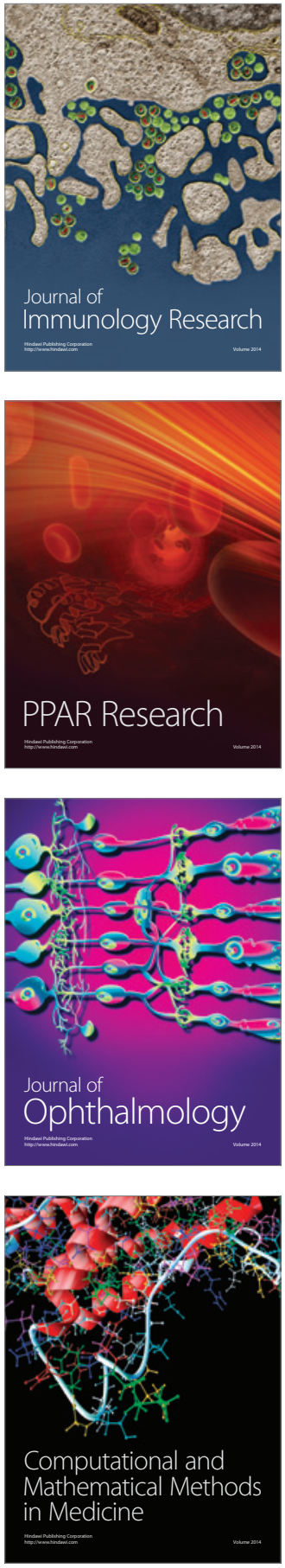

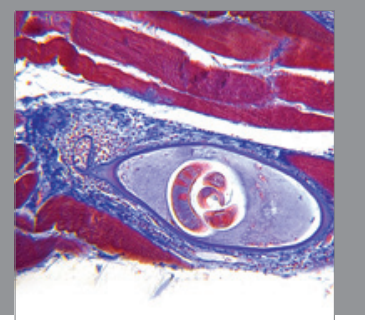

Gastroenterology

Research and Practice
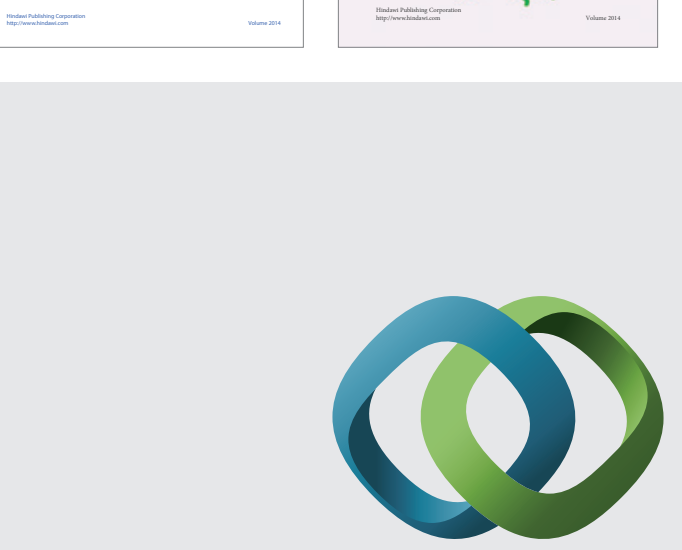

\section{Hindawi}

Submit your manuscripts at

http://www.hindawi.com
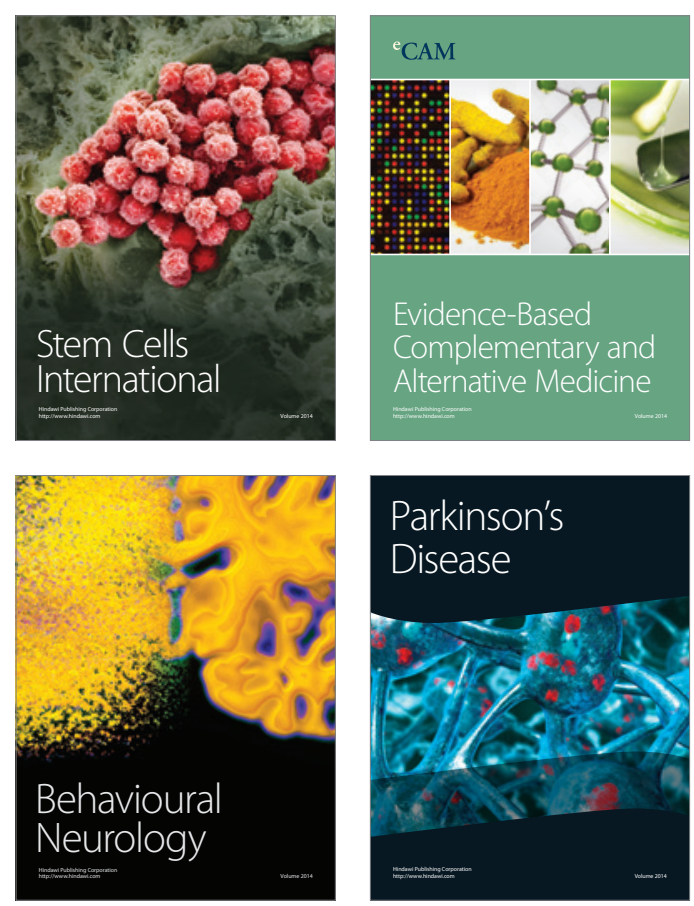

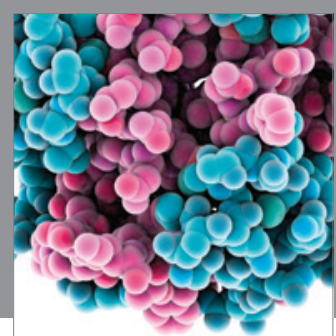

Journal of
Diabetes Research

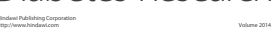

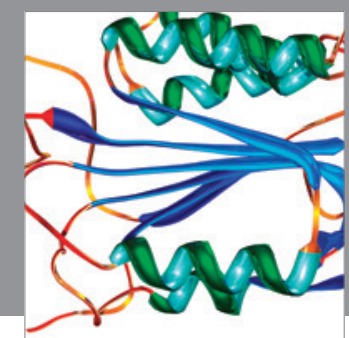

Disease Markers
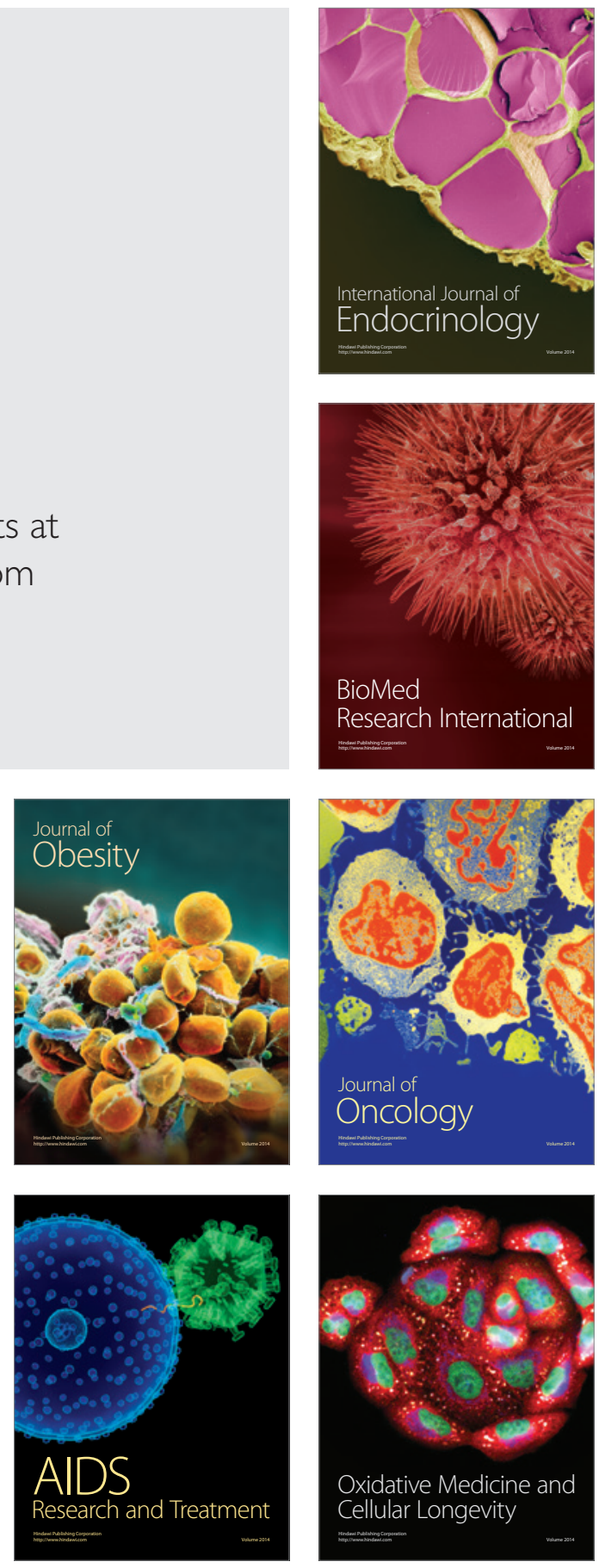